\title{
Unseen Word Representation by Aligning Heterogeneous Lexical Semantic Spaces
}

\author{
Victor Prokhorov, ${ }^{1}$ Mohammad Taher Pilehvar, ${ }^{1,2}$ Dimitri Kartsaklis, ${ }^{3 *}$ Pietro Liò, ${ }^{4}$ Nigel Collier ${ }^{1}$ \\ ${ }^{1}$ Department of Theoretical and Applied Linguistics, University of Cambridge \\ ${ }^{2}$ School of Computer Engineering, Iran University of Science and Technology, Tehran, Iran \\ ${ }^{3}$ Apple, Cambridge, UK \\ ${ }^{4}$ Department of Computer Science, University of Cambridge \\ vp361@cam.ac.uk, pilehvar@iust.ac.ir,dkartsaklis@apple.com,pl219@cam.ac.uk,nhc30@cam.ac.uk
}

\begin{abstract}
Word embedding techniques heavily rely on the abundance of training data for individual words. Given the Zipfian distribution of words in natural language texts, a large number of words do not usually appear frequently or at all in the training data. In this paper we put forward a technique that exploits the knowledge encoded in lexical resources, such as WordNet, to induce embeddings for unseen words. Our approach adapts graph embedding and cross-lingual vector space transformation techniques in order to merge lexical knowledge encoded in ontologies with that derived from corpus statistics. We show that the approach can provide consistent performance improvements across multiple evaluation benchmarks: in-vitro, on multiple rare word similarity datasets, and invivo, in two downstream text classification tasks.
\end{abstract}

\section{Introduction}

Word embeddings can be seamlessly integrated into various NLP systems, effectively enhancing their generalisation power (Camacho-Collados and Pilehvar 2018). However, the distributional approach to the semantic representation of words, either in its conventional count-based form or the recent neural-based paradigm, relies on a multitude of occurrences for each individual word to enable accurate representations. As a result, these corpus-based methods are unable to provide reliable representations for words that are infrequent or unseen during training, such as domain-specific terms. This is the case even if massive corpora are used for training, such as the Wikipedia corpus. ${ }^{1}$

To address the unseen word representation problem, several techniques have been proposed. Earlier works have mainly focused on morphologically complex words (Luong, Socher, and Manning 2013; Botha and Blunsom 2014; Soricut and Och 2015), whereas more recently, characterbased and subword unit information has garnered a lot of attention (Bojanowski et al. 2017). Despite their success, these

\footnotetext{
${ }^{*}$ Contributed to this work when he was with DTAL, Cambridge. Copyright (C) 2019, Association for the Advancement of Artificial Intelligence (www.aaai.org). All rights reserved.

${ }^{1}$ In the 2015 Wikipedia dump corpus with around 1.6B tokens, there are slightly over $1.9 \mathrm{M}$ word types with at least three occurrences. Of these word types, more than $80 \%$ appear at most 50 times in total, whereas more than two thirds of words in the vocabulary have frequency $\leq 20$.
}

models make two assumptions around the unseen word: (1) variations of the word exist in the training corpus (for instance, occurrences of track-or even untrack - should exist to induce embeddings for untracked); and (2) the semantics of the word can be estimated based on its subword units (which might not hold for single-morpheme words, e.g., galaxy, or for exocentric compounds, e.g., honeymoon). As a result, they fall short of effectively representing the semantics of unseen single-morpheme words for which no variation has been observed during training, essentially ignoring most of the rare domain-specific entities which are crucial for NLP systems when applied to those domains.

Furthermore, distributional techniques generally ignore the lexical knowledge encoded in dictionaries, ontologies, or other lexical resources. There exist hundreds of high coverage and domain-specific lexical resources which contain valuable information for infrequent words. Recently, various embedding induction techniques have attempted to leverage lexical resources, such as WordNet (Pilehvar and Collier 2017; Bahdanau et al. 2017) or Wikipedia (Lazaridou, Marelli, and Baroni 2017). Despite their success, they either rely on word definitions (glosses) or related words extracted from the the lexical resource while ignoring the knowledge encoded in the semantic structure. Here, we present a methodology that exploits the semantic structure of the lexical resource for unseen word representation. The technique first embeds a knowledge base into a vector space and then maps the embedded words from this space to a corpus-based space, in order to expand the vocabulary of the latter with additional representations for rare and unseen words. To our knowledge, it is the first time that vector space transformation techniques, which are widely used in multilingual settings, are leveraged for aligning heterogeneous monolingual spaces. We evaluate the reliability of our approach on several datasets across multiple tasks: six datasets for word similarity measurement and eight sentiment analysis and topic categorization datasets. Experimental results show that, unless ample occurrences exist in the training data, we can compute more reliable embeddings than the ones generated by state-of-the-art corpus based embedding techniques.

\section{Methodology}

Figure 1 illustrates our procedure for enriching an existing corpus vector space $\mathcal{S}_{\mathcal{C}}$ based on the lexical knowledge in an 


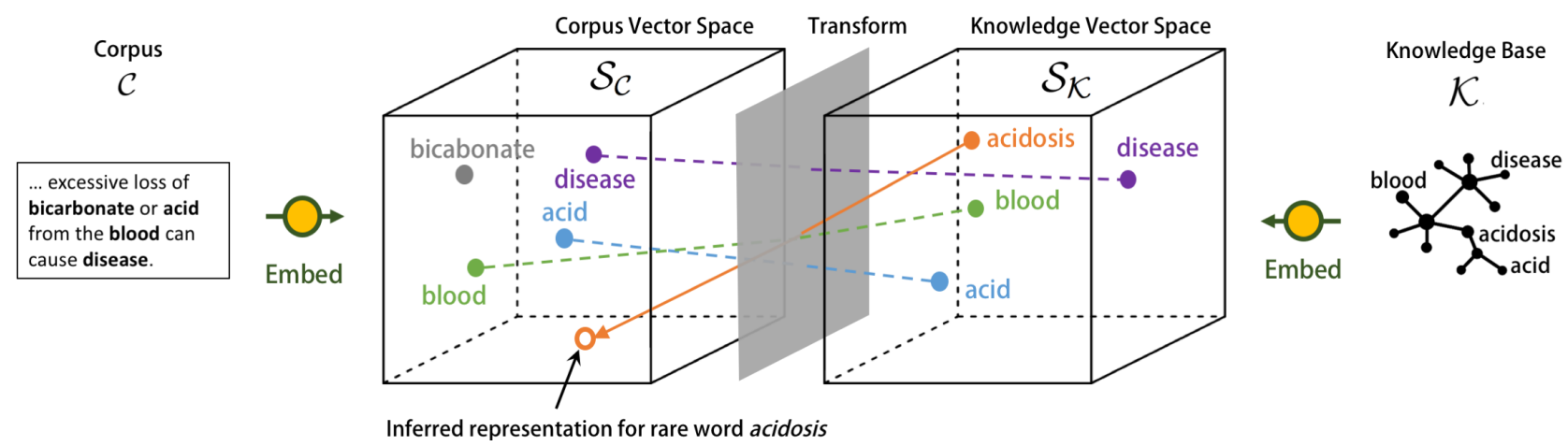

Figure 1: Our coverage enhancement procedure. The dashed lines represent semantic bridges and the solid line represents a rare word that is projected from the knowledge vector space to the corpus vector space.

external knowledge base $(\mathrm{KB}) \mathcal{K}$. The proposed algorithm mainly relies on techniques from two research areas: graph embedding and vector space transformation. Two main steps are involved in the process. First, it views $\mathcal{K}$ as a knowledge graph and transforms it to a vector space representation $\left(\mathcal{S}_{\mathcal{K}}\right)$ by leveraging graph embedding techniques $(\mathrm{Sec}-$ tion 2.2). Then, it aligns the two vector spaces, i.e., KB- $\left(\mathcal{S}_{\mathcal{K}}\right)$ and corpus-based $\left(\mathcal{S}_{\mathcal{C}}\right)$, by using vector space transformation algorithms (Section 2.3). As a result of this alignment, new embeddings are induced for unseen words in $\mathcal{S}_{\mathcal{C}}$. In our toy example in Figure 1, the term acidosis is missing from the vocabulary of $\mathcal{S}_{\mathcal{C}}$ but it is covered by the knowledge base $\mathcal{K}$. First, a graph embedding algorithm is used to embed $\mathcal{K}$, represented as a graph, into a vector space $\mathcal{S}_{\mathcal{K}}$. Then, based on common clues from the two spaces, a transformation function is learnt in order to map the vectors across the two spaces. The transformation function (from the embedded KB space $\mathcal{S}_{\mathcal{K}}$ to the corpus space $\mathcal{S}_{\mathcal{C}}$ ) allows us to project the vector for acidosis to the latter space, hence inducing a new representation for the word.

\subsection{Prerequisites}

The knowledge base embedding algorithms used in our procedure require $\mathcal{K}$ to be representable as a semantic network (knowledge graph). In our experiments, we used WordNet 3.0 (Fellbaum 1998) as external knowledge base. The resource contains around $120 \mathrm{~K}$ groups of synonyms, referred to as synsets, which are connected to each other by means of around $200 \mathrm{~K}$ lexical semantic relations, such as hypernymy and meronymy. Thanks to these relations, WordNet can be readily viewed as a semantic network. We further enrich the network by connecting a synset to all other synsets that appear in its disambiguated gloss ${ }^{2}$. This approach more than doubles the number of edges in WordNet's semantic network. As for the corpus vector space, any distributional semantic representation can be used. In our experiments, we opted mainly for word embeddings (rather than conventional count-based representations) due to their popularity.

Our procedure requires two additional conditions. Let $V_{\mathcal{K}}$ and $V_{\mathcal{C}}$ be the respective vocabularies of knowledge base and

\footnotetext{
${ }^{2}$ wordnet.princeton.edu/glosstag.shtml
}

corpus vector spaces. The first condition to be met is that $V_{\mathcal{K}}$ and $V_{\mathcal{C}}$ should have overlapping words, i.e., $V_{\mathcal{K}} \cap V_{\mathcal{C}} \neq \phi$. This is required for enabling the alignment of the two spaces (to be discussed in Section 2.3). The second condition is that the knowledge base $\mathcal{K}$ has to provide lexical knowledge for unseen or infrequent words in the corpus vector space. Thanks to the abundance of knowledge bases and the long tail of words in distributional representations, this condition is not difficult to be fulfilled.

\subsection{Knowledge Base Embedding}

The proposed coverage enhancement procedure starts by transforming the lexical knowledge representation in the knowledge base $\mathcal{K}$ to a form which is comparable to the corpus-based representation $\mathcal{S}_{\mathcal{C}}$. To this end, we embed the structural lexico-semantic knowledge of $\mathcal{K}$ into a vector space $\mathcal{S}_{\mathcal{K}}$.

We opted for node2 $\mathrm{vec}^{3}$ (Grover and Leskovec 2016), a random walk based graph embedding technique which has proven its potential in the reliable representation of graph nodes. Given a graph $G$, the algorithm first generates a stream of artificial "sentences" by performing a series of random walks over $G$. Each such "sentence" contains a sequence of "words" (i.e, vertices) such that consecutive words correspond to neighbouring vertices in $G$. Analogously to the natural language text in which semantically similar words are expected to appear in similar contexts, an artificial sentence encodes local information for a node from the graph by placing topologically close vertices in similar contexts. Representations are then computed for individual vertices by taking a similar objective to the Skip-gram model (Mikolov et al. 2013), i.e., by maximizing $\prod_{j=i-z, j \neq i}^{i+z} \operatorname{Pr}\left(w_{j} \mid w_{i}\right)$ which is the probability of a word $w_{i}$ given its context, where $z$ is the window size or the length of the random walk. The only difference from the original Skip-gram model lies in the way input "sentences" are constructed.

In our experiments, we set the parameters of node $2 \mathrm{vec}$ as follows: walk length to 100 , window size to 10 , and embed-

\footnotetext{
${ }^{3}$ https://github.com/snap-stanford/snap/tree/master/examples/ node $2 \mathrm{vec}$
} 
ding dimensionality to 100 . To decide on these parameters, we carried out experiments on the MTURK-771 dataset (Halawi et al. 2012). Also, note that nodes in the semantic graph of WordNet represent synsets. Hence, a polysemous word would correspond to multiple nodes. In our word similarity experiments (Sections 3.1 and 3.2) we use the MaxSim assumption of Reisinger and Mooney (2010) in order to map words to synsets: the similarity of two words is computed as that of their closest associated meanings. In the downstream experiment (Section 3.3), we compute a single word vector as the average of its corresponding synsets' vectors.

\subsection{Vector Space Alignment}

Once the lexical resource $\mathcal{K}$ is represented as a vector space $\mathcal{S}_{\mathcal{K}}$, we project it to $\mathcal{S}_{\mathcal{C}}$ in order to improve the word coverage of this space with additional words from $\mathcal{S}_{\mathcal{K}}$. In this procedure we make two assumptions. Firstly, the two spaces provide reliable models of word semantics; hence, the relative within-space distances between words in the two spaces are comparable. Secondly, there exists a set of shared words between the two spaces (also mentioned in Section 2.1); we refer to these words as semantic bridges.

For this transformation we opted for Canonical Correlation Analysis (Faruqui and Dyer 2014; Upadhyay et al. 2016, CCA), which is widely used for the projection of spaces belonging to different languages with the purpose of learning multilingual semantic spaces. ${ }^{4}$ The model receives as input two vector spaces for two different languages and a seed lexicon for that language pair, and learns a linear mapping between the two spaces. Ideally, words that are semantically similar across the two languages will be placed in close proximity to each other in the projected space.

Specifically, let $\mathcal{S}_{\mathcal{C}}^{\prime} \subset \mathcal{S}_{\mathcal{C}}$ and $\mathcal{S}_{\mathcal{K}}^{\prime} \subset \mathcal{S}_{\mathcal{K}}$ be the corresponding subsets of semantic bridges, i.e., words that are monosemous according to the WordNet sense inventory, for corpus and $\mathrm{KB}$ spaces, respectively. Note that $\mathcal{S}_{\mathcal{C}}^{\prime}$ and $\mathcal{S}_{\mathcal{K}}^{\prime}$ form matrices that contain representations for the same set of words, i.e., $\left|\mathcal{S}_{\mathcal{C}}^{\prime}\right|=\left|\mathcal{S}_{\mathcal{K}}^{\prime}\right|$. CCA finds a linear combination of dimensions in $\mathcal{S}_{\mathcal{C}}$ and $\mathcal{S}_{\mathcal{K}}$ which have maximum correlation with each other. Given two column vectors $\mathcal{S}_{\mathcal{C}}^{\prime}$ and $\mathcal{S}_{\mathcal{K}}^{\prime}$ of embeddings in the two spaces, CCA computes vectors $w_{\mathcal{C}}$ and $w_{\mathcal{K}}$ such that the random variables $w_{\mathcal{C}} \mathcal{S}_{\mathcal{C}}^{\prime}$ and $w_{\mathcal{K}} \mathcal{S}_{\mathcal{K}}^{\prime}$ maximize the correlation $\rho\left(w_{\mathcal{C}} \mathcal{S}_{\mathcal{C}}^{\prime}, w_{\mathcal{K}} \mathcal{S}_{\mathcal{K}}^{\prime}\right)$ :

$$
\begin{aligned}
w_{\mathcal{C}}^{*}, w_{\mathcal{K}}^{*} & =\operatorname{CCA}\left(\mathcal{S}_{\mathcal{K}}^{\prime}, \mathcal{S}_{\mathcal{C}}^{\prime}\right) \\
& =\underset{w_{\mathcal{C}}, w_{\mathcal{K}}}{\arg \max } \rho\left(w_{\mathcal{C}} \mathcal{S}_{\mathcal{C}}^{\prime}, w_{\mathcal{K}} \mathcal{S}_{\mathcal{K}}^{\prime}\right) \\
& =\underset{w_{\mathcal{C}}, w_{\mathcal{K}}}{\arg \max } \frac{w_{\mathcal{C}} \Sigma_{\mathcal{C} \mathcal{K}} w_{\mathcal{K}}}{\sqrt{w_{\mathcal{C}} \Sigma_{\mathcal{C}} w_{\mathcal{C}}^{T}} \sqrt{w_{\mathcal{K}} \Sigma_{\mathcal{K}} w_{\mathcal{K}}^{T}}}
\end{aligned}
$$

where $\Sigma_{X}$ and $\Sigma_{X, Y}$ denote covariance and crosscovariance, respectively. Note that the maximization is invariant to scaling of $w_{\mathcal{C}}$ and $w_{\mathcal{K}}$. Hence, we can have a constraint for unit variance:

\footnotetext{
${ }^{4} \mathrm{We}$ also performed experiments with least squares regression (Mikolov, Le, and Sutskever 2013; Dinu and Baroni 2014). However, CCA proved to be consistently better. We do not report LS results due to lack of space.
}

$$
w_{\mathcal{C}}^{*}, w_{\mathcal{K}}^{*}=\underset{w_{\mathcal{C}} \Sigma_{\mathcal{C}} w_{\mathcal{C}}^{T}=w_{\mathcal{K}} \Sigma_{\mathcal{K}} w_{\mathcal{K}}^{T}=1}{\arg \max } w_{\mathcal{C}} \Sigma_{\mathcal{C} \mathcal{K}} w_{\mathcal{K}}
$$

The dimensionality of the resultant space in our experiments is $\min \left(d_{\mathcal{C}}, d_{\mathcal{K}}\right)=d_{\mathcal{K}}=100$, where $d_{\mathcal{C}}$ and $d_{\mathcal{K}}$ are the dimensionalities of the corpus and $\mathrm{KB}$ spaces, respectively. An additional constraint forces these projections to be uncorrelated. The enhanced space $\mathcal{S}^{*}$ is obtained as the union of $w_{\mathcal{C}} \mathcal{S}_{\mathcal{C}}$ and $w_{\mathcal{K}} \mathcal{S}_{\mathcal{K}}$. Note that this procedure is slightly different from the one illustrated in Figure 1. The enriched space is a third space which is independent from the two initial spaces $\mathcal{S}_{\mathcal{K}}$ and $\mathcal{S}_{\mathcal{C}}$

As for the seed lexicon (the set of semantic bridges $\mathcal{S}_{\mathcal{C}}^{\prime}$ and $\mathcal{S}_{\mathcal{K}}^{\prime}$ ), we used the set of monosemous words in the WordNet's vocabulary which are deemed to have the most reliable semantic representations in the corpus vector space. Of the $155 \mathrm{~K}$ words in WordNet's vocabulary, around $128 \mathrm{~K}$ are monosemous, which provides us with a large set of semantic bridges to use for the alignment step. However, in our experiments we found that a small subset of $5 \mathrm{~K}$ semantic bridges is enough for achieving reliable transformations.

Graph embedding and space alignment. For this work we experimented with node2vec. We note that there is a rich literature for graph embeddings (Cai, Zheng, and Chang 2017). A series of algorithms first construct an adjacency matrix of the graph and obtain embeddings by directly factorising this matrix (Cao, Lu, and Xu 2015; Roweis and Saul 2000), whereas others employ deep learning techniques, such as autoencoders (Wang, Cui, and Zhu 2016). Relation embedding techniques such as TransE (Bordes et al. 2013) and HOLE (Nickel, Rosasco, and Poggio 2016) are not suitable candidates for our purpose since their focus is rather on the embedding of edges (as opposed to nodes). As noted before, for the space alignment we experimented with CCA which is a linear model of projection. We leave the evaluation of non-linear transformation techniques, such as Kernel CCA (Akaho 2006) and Deep CCA (Andrew et al. 2013), and other graph embedding techniques, to future work.

\section{Experiments}

In this section we provide three different sets of experiments that were carried out to evaluate the reliability of our rare word embedding induction technique (which we will refer to as AligN). First, we report results for in-vitro evaluations on the Stanford Rare Word similarity dataset (Section 3.1 ) and in a simulated rare word similarity setting (Section 3.2). We then verify the reliability of our induced embeddings in two downstream NLP tasks, sentiment analysis and topic categorization. This experiment is detailed in Section 3.3. The code used in our experiments will be released to allow future experimentation and comparison. ${ }^{5}$

\subsection{Rare Word Similarity}

The Stanford Rare Word (RW) Similarity dataset (Luong, Socher, and Manning 2013) has been regarded as a standard benchmark for evaluating embedding induction techniques.

\footnotetext{
${ }^{5}$ https://github.com/VictorProkhorov/AAAI2019
} 


\begin{tabular}{lcclcc}
\hline \multirow{2}{*}{ Embedding } & \multicolumn{2}{c}{ W2 V-GN } & & \multicolumn{2}{c}{ W2V-WP } \\
\cline { 2 - 3 } \cline { 6 - 7 } & $r$ & $\rho$ & & $r$ & $\rho$ \\
\hline W2V-GN & 0.44 & 0.45 & & 0.41 & 0.43 \\
+ Additive & 0.46 & 0.48 & & 0.41 & 0.43 \\
+ SemLand & 0.48 & 0.51 & & 0.39 & 0.40 \\
+ LSTM & 0.48 & 0.50 & & 0.40 & 0.40 \\
+ ALIGN & 0.48 & 0.48 & & 0.42 & 0.42 \\
\hline
\end{tabular}

Table 1: Pearson $(r)$ and Spearman $(\rho)$ correlation for our approach (ALIGN) on the RW dataset with two pre-trained sets of word embeddings, before and after enhancement with various methods. FastText-WP (trained on the Wikipedia corpus): $r=0.44, \rho=0.44$ and node 2 vec (without any alignment and independent from corpus embeddings): $r=0.16$, $\rho=0.16$.

The dataset comprises 2034 pairs of infrequent words, such as ulcerate-change and nurturance-care. In the first evaluation, we use this benchmark to compare our model against recent rare word representation techniques.

Experimental setup. We experimented with two sets of word2vec (Mikolov et al. 2013) embeddings trained on two different corpora: (1) W2V-GN, the Google News (vocab: $3 \mathrm{M}$, dim: 300$)^{6}$, and (2) W2 V-WP, the Wikipedia corpus (Shaoul and Westbury 2010) (vocab: 2.4M, dim: 300). As for comparison systems, we benchmark our results against four other approaches: (1) SemLand (Pilehvar and Collier 2017) which extracts for an unseen word the set of its semantically related words from WordNet and induces an embedding by combining their embeddings; (2) the Additive model of Lazaridou, Marelli, and Baroni (2017) which takes the unseen word's definition as semantic clue and induces an embedding by adding (averaging) the embeddings of content words in the defintion; (3) LSTM-based strategy of Bahdanau et al. (2017) which is a more complex version of the additive model that relies on an LSTM network which receives as its input the WordNet definition of the unseen word; and (4) Fast Text (Bojanowski et al. 2017) which computes a word embedding by combining the embeddings of its sub-word character n-grams (see Section 4 for more details).

Results. Table 1 shows correlation performance on the dataset for the two pre-trained word embeddings, in their initial form and when enhanced with additional induced word embeddings. Among the two initial embeddings, w2 V-GN provides a lower coverage (173 out-of-vocabulary words vs. 88 for $\mathrm{w} 2 \mathrm{~V}-\mathrm{WP}$ ) despite its larger vocabulary (3M vs. 2.4M). All enhanced embeddings attain near full coverage (over 99\%), thanks to the vocabulary expansion offered by WordNet. Our approach (ALIGN) produces competitive performance across the two settings and according to both Pearson and Spearman correlation metrics. The performance

\footnotetext{
${ }^{6}$ code.google.com/archive/p/word2vec/
}

$(r=0.16, \rho=0.16)$ of node2vec, when independently applied to this dataset, is notably lower than that of the initial corpus embeddings. However, it is interesting to note that these non-optimal embeddings can better the performance of corpus embeddings when combined with them, showing the complementarity of the two sources of information.

Comparison with FastText. FastText proves competitive on the dataset ( $r=0.44, \rho=0.44)$, highlighting the effectiveness of induced word embeddings from sub-word (character) information. This is not a surprise given that around a third of the rare words in the RW dataset are plural or $e d$ forms which can be easily handled by resorting to the embedding of their singular or uninflected forms. For instance, kindergarteners and postponements are highly similar to their singular forms and the semantics of encrusted and entrapped can be estimated to a good extent from encrust and entrap which are relatively more frequent terms. None of the other models in the table have access to this information. However, as mentioned earlier, the sub-word backoff strategy might not be effective for single-morpheme words and exocentric compounds, which in a real-world scenario account for the most frequent cases of unseen words and can be effectively handled by our model.

Reliability of the RW dataset. The Stanford Rare Word Similarity dataset has been regarded as a standard evaluation benchmark for rare word representation and similarity, and as such it is included in the experiments of this paper. However, the variance across the scores provided by different annotators for the same pair is generally high in this dataset. This is mainly due to the reliance of the dataset on crowdsourcing without having rigorous checkpoint on the raters. As also highlighted by Pilehvar et al. (2018), the lowconfidence annotations are also reflected by contradictory instances, such as the two (almost) identical pairs tricolourflag and tricolor-flag which have received the two very different scores of 5.80 and 0.71 . Hence, further improvements on the dataset (over the $\mathrm{W} 2 \mathrm{~V}-\mathrm{GN}$ baseline), provided by different techniques, cannot be meaningfully interpreted. Given the unreliability of the benchmark, in the following section, we provide an alternative evaluation based on standard (common) word similarity benchmarks.

\subsection{Simulated Rare Word Similarity}

For a word similarity dataset to be suitable for this evaluation, it has to contain words that are infrequent in generic texts. However, most of the existing standard word similarity datasets contain only high frequency words, which makes them unsuitable for evaluating rare word representation techniques. To work around this limitation, we follow Sergienya and Schütze (2015) and leverage corpus downsampling in order to artificially transform standard word similarity datasets to rare word similarity benchmarks. This enables us to evaluate our embedding induction technique on a variety of standard datasets. 


\begin{tabular}{|c|c|c|c|c|c|c|c|c|c|c|c|}
\hline \multirow{2}{*}{ Embedding } & \multirow{2}{*}{ Setting } & \multicolumn{2}{|c|}{ RG-65 } & \multicolumn{2}{|c|}{ SimLex-999 } & \multicolumn{2}{|c|}{ MEN-3000 } & \multicolumn{2}{|c|}{ SimVerb-3500 } & \multicolumn{2}{|c|}{ WS-353 Sim } \\
\hline & & $r$ & $\rho$ & $r$ & $\rho$ & $r$ & $\rho$ & $r$ & $\rho$ & $r$ & $\rho$ \\
\hline \multirow{4}{*}{ Initial word2vec } & $T=10$ & 0.40 & 0.42 & 0.15 & 0.12 & 0.46 & 0.45 & 0.07 & 0.08 & 0.53 & 0.54 \\
\hline & $T=20$ & 0.54 & 0.56 & 0.22 & 0.21 & 0.53 & 0.52 & 0.12 & 0.11 & 0.63 & 0.62 \\
\hline & $T=50$ & 0.63 & 0.63 & 0.26 & 0.24 & 0.63 & 0.62 & 0.15 & 0.15 & 0.68 & 0.69 \\
\hline & $T=100$ & 0.68 & 0.69 & 0.30 & 0.28 & 0.65 & 0.64 & 0.19 & 0.18 & 0.73 & 0.73 \\
\hline ALIGN & $T=0$ & 0.86 & 0.88 & 0.40 & 0.37 & 0.65 & 0.66 & 0.42 & 0.39 & 0.71 & 0.69 \\
\hline LSTM & $T=0$ & 0.52 & 0.57 & 0.19 & 0.19 & 0.19 & 0.20 & 0.28 & 0.29 & 0.18 & 0.21 \\
\hline Additive & $T=0$ & 0.56 & 0.59 & 0.17 & 0.13 & 0.24 & 0.23 & 0.21 & 0.20 & 0.31 & 0.32 \\
\hline SemLand & $T=0$ & 0.52 & 0.53 & 0.22 & 0.20 & 0.38 & 0.38 & 0.23 & 0.22 & 0.43 & 0.40 \\
\hline FastText & $T=0$ & 0.77 & 0.80 & 0.32 & 0.32 & 0.76 & 0.76 & 0.22 & 0.21 & 0.74 & 0.73 \\
\hline
\end{tabular}

Table 2: Results of corpus-based and enhanced embeddings in the simulated rare word similarity setting.

Experimental setup. Let $T$ be the rarity threshold, i.e., the expected occurrence frequency of an artificial rare word in the training text corpus. We process the original text corpus in order to guarantee that each word in the similarity dataset appears at most $T$ times in the training corpus. This can be achieved by replacing all but $T$ occurrences of the word with another unique token (e.g., the word concatenated by some unique character). As a result of this procedure, we obtain a corpus for each $T$ value and for each dataset. Training word embeddings on these corpora simulates a setting in which all the words in the word similarity dataset are rare as they occur infrequently in the training corpus. Except from the corpus downsampling step, the experimental setup is similar to that of the previous experiment.

Datasets. For this experiment, we opted for five standard word similarity datasets: RG-65 (Rubenstein and Goodenough 1965), SimLex-999 (Hill, Reichart, and Korhonen 2015), MEN (Bruni, Tran, and Baroni 2014), WordSim-353 similarity subset (Agirre et al. 2009), and SimVerb-3500 (Gerz et al. 2016) which contains verbs only.

Results. Table 2 lists correlation performance results on the five datasets and for four different values of $T(10,20$, 50 , and 100) for the initial downsampled W2V-WP embeddings ${ }^{7}$ as well as for enhanced embeddings using different techniques for $T=0$ (unseen word setting). As expected, there is a steady improvement for the corpus-based embeddings with increasing values of $T$. On all the datasets and according to both evaluation measures, ALIGN significantly improves over the three other WordNet-based approaches. Interestingly, our induced embeddings consistently outperform corpus embeddings which are constructed with $T=$ 10,20 , and 50 on all the datasets and are often better or on par with $T=100$. This means that our approach can produce embeddings that are as reliable as those corpus embeddings that are computed based on 100 occurrences. This is important as around $80 \%$ of the words in the vocabulary

\footnotetext{
${ }^{7}$ Obviously, for $T=0$, word2vec would be unable to learn any embeddings, hence we do not show that setting.
}

of the Wikipedia corpus appear fewer than 50 times in the whole corpus (see Footnote 1). Moreover, surprisingly, on the SimVerb dataset the induced embeddings perform significantly better than the corpus-based embeddings, even at $T=100$. This shows the superior quality of the induced verb embeddings, thanks to the hand-crafted part-of-speechspecific knowledge encoded for them in WordNet.

Similarly to the previous experiment, FastText proves to be a competitive baseline, outperforming our induced embeddings on two datasets. However, again, we note that FastText benefits from the advantage of having access to all plural forms of these (originally frequent) downsampled words in the training dataset, which might not establish a fair comparison. The simulated rare word similarity datasets address the unreliability issue of Stanford RW but still do not represent a real-world rare word scenario. Ideally, such a dataset would contain named entities, domain-specific terms or other uncommon words that tend to appear infrequently in generic text corpora (which are often used for training word embeddings). We believe that rare word representation research requires such a high quality benchmark for more rigorous evaluations. We leave the possibility of the creation of such datasets to future work.

\subsection{Evaluation in Downstream Tasks}

We were also interested in having an in-vivo evaluation of the reliability of our induced embeddings in a real-world NLP system. Given that currently the most important application of word embeddings is in the initialization of the input layer in neural networks, we opted for a standard neural system as our evaluation benchmark.

Experimental setup. We experimented with a neural text classification system applied to two tasks: sentiment analysis (binary classification) and topic categorization (multi-class classification). The embedding layer of this system is initialized with pre-trained word2vec embeddings. Let $L$ be the vocabulary of a given dataset. We dropped the pre-trained corpus embeddings for $X \%$ of the words in $L$ and replaced them with our induced embeddings. We experimented with three $X$ values: 0 (in which we used all the corpus embed- 


\begin{tabular}{|c|c|c|c|c|c|c|c|c|c|}
\hline \multirow{2}{*}{ Initialization } & \multirow{2}{*}{ Setting } & \multicolumn{5}{|c|}{ Sentiment Analysis } & \multicolumn{3}{|c|}{ Topic Categorization } \\
\hline & & PL04 & PL05 & RTC & IMDB & Stanford & $\mathrm{BBC}$ & NG & $\mathrm{OH}$ \\
\hline \multirow{2}{*}{$X=0 \%$} & Initial & 66.2 & 75.4 & 79.7 & 85.4 & 80.4 & 96.7 & 86.5 & 27.8 \\
\hline & + AligN & 63.7 & 75.6 & 79.4 & 86.8 & 80.5 & 96.5 & 87.0 & 29.3 \\
\hline \multirow{2}{*}{$X=20 \%$} & Initial & 59.1 & 67.2 & 63.8 & 71.1 & 70.1 & 93.1 & 67.4 & 16.4 \\
\hline & + AligN & 58.9 & 69.9 & 74.5 & 79.3 & 77.6 & 95.1 & 80.3 & 25.7 \\
\hline \multirow{2}{*}{$X=40 \%$} & Initial & 56.2 & 63.5 & 62.7 & 70.3 & 66.1 & 91.0 & 62.8 & 15.7 \\
\hline & + ALIGN & 55.6 & 68.0 & $\mathbf{7 4 . 5}$ & 81.8 & 76.2 & 94.5 & 79.7 & 28.5 \\
\hline
\end{tabular}

Table 3: Accuracy performance on eight datasets for sentiment analysis and topic categorization. The best results for each setting are shown in bold. NG and OH stand for Newsgroups and Ohsumed, respectively.

dings to initialize the layer; new embeddings were induced to further improve coverage for those words missing in corpus embeddings' vocabulary), 20 and 40 (in which, respectively, $20 \%$ and $40 \%$ of corpus embeddings were dropped, i.e., their corresponding words were treated as out of vocabulary). We were mainly interested in observing if the induced embeddings, first, could improve over corpus embeddings and, second, were able to re-gain system performance lost when dropping a part of the corpus embeddings. In all settings the embedding layer was not updated during training (static). This allows us to have a direct evaluation on the reliability of embeddings, independently from any updates and alteration they can undergo during training. In each configuration we repeat the experiment three times and report the average performance.

Text classification system. In our experiments, we used a CNN text classifier which is similar to that of Kim (2014). The only difference is that in our model, instead of directly inputting the pooled features from the convolutional layer to a fully connected softmax layer, they are first passed through a recurrent layer in order to enable a better capturing of longdistance dependencies. Specifically, as our recurrent layer we used LSTM (Hochreiter and Schmidhuber 1997).

Datasets. For sentiment analysis we used five standard datasets, including PL04 (Pang and Lee 2004), PL05 (Pang and Lee 2005), ${ }^{8} \mathrm{RTC}^{9}$, and IMDB (Maas et al. 2011) which are all binary datasets (with positive and negative labels) containing snippets of or full movie reviews. We also experimented with Stanford Sentiment dataset (Socher et al. 2013) which associates phrases with values that denotes their sentiments. To be consistent with the other four datasets' binary classification setting, we removed the neutral phrases with scores 0.4 to 0.6 and considered the reviews with values below 0.4 as negative and above 0.6 as positive. For the topic categorization task we used two newswire datasets: The BBC news dataset CR ${ }^{10}$ (Greene and Cunningham

\footnotetext{
${ }^{8}$ Both PL04 and PL05 are obtained from http://www.cs.cornell. edu/people/pabo/movie-review-data/

${ }^{9} \mathrm{http}: / / \mathrm{www}$. rottentomatoes.com

${ }^{10} \mathrm{http} / / / \mathrm{mlg} \cdot \mathrm{ucd} . \mathrm{ie} /$ datasets/bbc.html
}

2006) and Newsgroups (Lang 1995) with 5 and 20 classes, respectively. We also experimented with a domain-specific categorization dataset: Ohsumed ${ }^{11}$, which contains medical texts categorized into 23 classes.

Results. Table 3 shows the results. We report classification accuracy for the baseline system ("Initial") which is initialized by full $(X=0 \%)$ or partial $(X>0 \%)$ corpus-based embeddings, and for the enhanced systems with additional induced embeddings ("+ALIGN"). Generally, the enhancement proves to be beneficial as it provides improvements in most of the configurations across the eight datasets. In the $X=0 \%$ setting, the improvement is particularly noticeable for the IMDB, Newsgroup and Ohsumed datasets which have a fair portion of their vocabularies not covered by word2vec embeddings. However, lower or no improvement is observed for other datasets (particularly, PL04) whose vocabularies are largely covered by the corpus embeddings. In the $X>0 \%$ settings, the performance of the baseline system drops significantly on most datasets. In the $20 \%$ setting, which is the closest to a real-world scenario, the enhanced system can recover a large part of the lost performance on most of the datasets. The same trend is observed for $X=40 \%$. Interestingly, on the Ohsumed dataset, which belongs to the medical domain, the enhanced system gets close to the initial system initialized by corpus embeddings. This is a strong indication of the effectiveness of our approach in filling lexical gaps for specific domains. Overall, the results show that our induced embeddings, though not sufficient to replace corpus embeddings for frequent words, can significantly improve over infrequent or unkown embeddings, particularly for specific domains.

\section{Related Work}

Given its importance, unseen word representation has attracted considerable research attention for the past few years. Earlier techniques have mainly focused on improving distributional models for better handling of infrequent words (Sergienya and Schütze 2015), or on inducing embeddings for morphological variations (Alexandrescu and Kirchhoff 2006; Luong, Socher, and Manning 2013; Lazaridou et al.

\footnotetext{
${ }^{11} \mathrm{ftp}: / /$ medir.ohsu.edu/pub/ohsumed
} 
2013; Botha and Blunsom 2014; Soricut and Och 2015). The latter branch often utilizes a morphological segmenter, such as Morfessor (Creutz and Lagus 2007), in order to break inflected words into their components and to compute representations by extending the semantics of an unseen word's morphological variations.

More recently, character-based models have garnered a lot of attention. In these models words are broken down into subword units and characters (Bojanowski et al. 2017), usually irrespective of their morphological structure. An unseen word's representation is induced by combining the information for its subword units; for instance, by averaging the vector representations of its constituent character n-grams as done by FastText (Bojanowski et al. 2017). Character-based models have been successfully tested in different NLP tasks, including language modeling (Sutskever, Martens, and Hinton 2011; Graves 2013), part-of-speech tagging (Dos Santos and Zadrozny 2014; Ling et al. 2015) and syntactic parsing (Ballesteros, Dyer, and Smith 2015). However, all these techniques fall short of inducing representations for singlemorpheme words that are not seen frequently during training as they base their modeling on information available from sub-word units. In contrast, our alignment-based model can also induce embeddings for single-morpheme words that are infrequent or unseen in the training data, such as domainspecific entities.

Most related to our work are the WordNet-based approaches of Pilehvar and Collier (2017) and Bahdanau et al. (2017). The former computes an unseen word's embedding by extracting the set of its semantically similar words ("semantic landmarks") from WordNet and combining their embeddings, whereas the latter trains a recurrent neural network, specifically, an LSTM, to estimate a word's embedding given its definition from WordNet. Moreover, the additive model of Lazaridou, Marelli, and Baroni (2017) is analoguous to the LSTM model (though less complex) and computes an embedding as the centroid of the embedding of the words in its definition. Despite addressing the singlemorpheme word representation limitation of morphological models, these approaches ignore the information encoded in WordNet's lexical-semantic relations. We improve over these by proposing a model that effectively leverages the semantic network of WordNet. Our experimental results show that, for the task of embedding induction, structural information can result in a more consistent performance than glosses or similar words.

\section{Conclusions and Future Work}

We presented a methodology for marrying distributional semantic spaces with lexical knowledge bases and applied it to the task of extending the vocabulary of the former with the help of information extracted from the latter. We showed the reliability of our approach by evaluating the induced embeddings on multiple word similarity benchmarks as well as on a downstream NLP evaluation framework. In future work, we plan to experiment with domain-specific lexical resources, such as medical ontologies, and study the efficacy of our methodology on adapting downstream NLP systems to new domains.

\section{Acknowledgments}

We would like to thank the anonymous reviewers for their comments. This research was supported by an EPSRC Experienced Researcher Fellowship (N. Collier, D. Kartsaklis: EP/M005089/1) and an MRC grant (M.T. Pilehvar: MR/M025160/1). We gratefully acknowledge the donation of a GPU from the NVIDIA Grant Program.

\section{References}

Agirre, E.; Alfonseca, E.; Hall, K.; Kravalova, J.; Paşca, M.; and Soroa, A. 2009. A study on similarity and relatedness using distributional and WordNet-based approaches. In Proceedings of HLT-NAACL, 19-27.

Akaho, S. 2006. A kernel method for canonical correlation analysis. CoRR abs/cs/0609071.

Alexandrescu, A., and Kirchhoff, K. 2006. Factored neural language models. In Proceedings of HLT-NAACL, 1-4.

Andrew, G.; Arora, R.; Livescu, K.; and Bilmes, J. 2013. Deep canonical correlation analysis. In Proceedings of ICML.

Bahdanau, D.; Bosc, T.; Jastrzebski, S.; Grefenstette, E.; Vincent, P.; and Bengio, Y. 2017. Learning to compute word embeddings on the fly. CoRR abs/1706.00286.

Ballesteros, M.; Dyer, C.; and Smith, N. A. 2015. Improved transition-based parsing by modeling characters instead of words with lstms. In Proceedings of EMNLP, 349-359.

Bojanowski, P.; Grave, E.; Joulin, A.; and Mikolov, T. 2017. Enriching word vectors with subword information. Transactions of the Association for Computational Linguistics 5:135-146.

Bordes, A.; Usunier, N.; Garcia-Durán, A.; Weston, J.; and Yakhnenko, O. 2013. Translating embeddings for modeling multi-relational data. In Proceedings of the 26th International Conference on Neural Information Processing Systems - Volume 2, NIPS'13, 2787-2795.

Botha, J. A., and Blunsom, P. 2014. Compositional Morphology for Word Representations and Language Modelling. In Proceedings of ICML, 1899-1907.

Bruni, E.; Tran, N. K.; and Baroni, M. 2014. Multimodal distributional semantics. JAIR 49(1):1-47.

Cai, H.; Zheng, V. W.; and Chang, K. C. 2017. A comprehensive survey of graph embedding: Problems, techniques and applications. CoRR abs/1709.07604.

Camacho-Collados, J., and Pilehvar, M. T. 2018. From word to sense embeddings: A survey on vector representations of meaning. Journal of Artificial Intelligence Research.

Cao, S.; Lu, W.; and Xu, Q. 2015. Grarep: Learning graph representations with global structural information. In Proceedings of CIKM, 891-900.

Creutz, M., and Lagus, K. 2007. Unsupervised models for morpheme segmentation and morphology learning. ACM Trans. on Speech and Language Processing 4(1):3:1-3:34.

Dinu, G., and Baroni, M. 2014. Improving zeroshot learning by mitigating the hubness problem. CoRR abs/1412.6568. 
Dos Santos, C. N., and Zadrozny, B. 2014. Learning character-level representations for part-of-speech tagging. In Proceedings of ICML, II-1818-II-1826.

Faruqui, M., and Dyer, C. 2014. Improving vector space word representations using multilingual correlation. In Proceedings of EACL, 462-471.

Fellbaum, C., ed. 1998. WordNet: An Electronic Database. Cambridge, MA: MIT Press.

Gerz, D.; Vulić, I.; Hill, F.; Reichart, R.; and Korhonen, A. 2016. Simverb-3500: A large-scale evaluation set of verb similarity. In Proceedings of EMNLP, 2173-2182.

Graves, A. 2013. Generating sequences with recurrent neural networks. CoRR abs/1308.0850.

Greene, D., and Cunningham, P. 2006. Practical solutions to the problem of diagonal dominance in kernel document clustering. In Proceedings of ICML, 377-384. ACM.

Grover, A., and Leskovec, J. 2016. Node2vec: Scalable feature learning for networks. In Proceedings of KDD, 855864.

Halawi, G.; Dror, G.; Gabrilovich, E.; and Koren, Y. 2012. Large-scale learning of word relatedness with constraints. In Proceedings of KDD, 1406-1414.

Hill, F.; Reichart, R.; and Korhonen, A. 2015. SimLex999: Evaluating semantic models with (genuine) similarity estimation. Computational Linguistics 41(4):665-695.

Hochreiter, S., and Schmidhuber, J. 1997. Long short-term memory. Neural computation 9(8):1735-1780.

Kim, Y. 2014. Convolutional neural networks for sentence classification. In Proceedings of EMNLP, 1746-1751.

Lang, K. 1995. Newsweeder: Learning to filter netnews. In Proceedings of ICML, 331-339.

Lazaridou, A.; Marelli, M.; Zamparelli, R.; and Baroni, M. 2013. Compositional-ly derived representations of morphologically complex words in distributional semantics. In Proceedings of ACL, 1517-1526.

Lazaridou, A.; Marelli, M.; and Baroni, M. 2017. Multimodal word meaning induction from minimal exposure to natural text. Cognitive Science 41(S4):677-705.

Ling, W.; Dyer, C.; Black, A. W.; Trancoso, I.; Fermandez, R.; Amir, S.; Marujo, L.; and Luis, T. 2015. Finding function in form: Compositional character models for open vocabulary word representation. In Proceedings of EMNLP, 1520-1530.

Luong, T.; Socher, R.; and Manning, C. 2013. Better word representations with recursive neural networks for morphology. In Proceedings of CoNLL, 104-113.

Maas, A. L.; Daly, R. E.; Pham, P. T.; Huang, D.; Ng, A. Y.; and Potts, C. 2011. Learning word vectors for sentiment analysis. In Proceedings of ACL-HLT, 142-150.

Mikolov, T.; Chen, K.; Corrado, G.; and Dean, J. 2013. Efficient estimation of word representations in vector space. In Workshop at ICLR.

Mikolov, T.; Le, Q. V.; and Sutskever, I. 2013. Exploiting similarities among languages for machine translation. CoRR abs/1309.4168.
Nickel, M.; Rosasco, L.; and Poggio, T. 2016. Holographic embeddings of knowledge graphs. In Proceedings of the Thirtieth AAAI Conference on Artificial Intelligence, AAAI'16, 1955-1961.

Pang, B., and Lee, L. 2004. A sentimental education: Sentiment analysis using subjectivity summarization based on minimum cuts. In Proceedings of ACL, 51-61.

Pang, B., and Lee, L. 2005. Seeing stars: Exploiting class relationships for sentiment categorization with respect to rating scales. In Proceedings of ACL, 115-124.

Pilehvar, M. T., and Collier, N. 2017. Inducing embeddings for rare and unseen words by leveraging lexical resources. In Proceedings of the EACL, 388-393.

Pilehvar, M. T.; Kartsaklis, D.; Prokhorov, V.; and Collier, N. 2018. Card-660: Cambridge rare word dataset - a reliable benchmark for infrequent word representation models. In Proceedings of EMNLP.

Reisinger, J., and Mooney, R. J. 2010. Multi-prototype vector-space models of word meaning. In Proceedings of HLT-NAACL, 109-117.

Roweis, S. T., and Saul, L. K. 2000. Nonlinear Dimensionality Reduction by Locally Linear Embedding. Science 290(5500):2323-2326.

Rubenstein, H., and Goodenough, J. B. 1965. Contextual correlates of synonymy. Communications of the ACM 8(10):627-633.

Sergienya, I., and Schütze, H. 2015. Learning better embeddings for rare words using distributional representations. In Proceedings of EMNLP, 280-285.

Shaoul, C., and Westbury, C. 2010. The Westbury Lab Wikipedia Corpus. http://www.psych.ualberta.ca/ $\sim$ westburylab/downloads/westburylab.wikicorp.download. html. Accessed: 2016-11-10.

Socher, R.; Perelygin, A.; Wu, J.; Chuang, J.; Manning, C.; $\mathrm{Ng}, \mathrm{A}$;; and Potts, C. 2013. Parsing with compositional vector grammars. In Proceedings of EMNLP, 455-465.

Soricut, R., and Och, F. 2015. Unsupervised morphology induction using word embeddings. In Proceedings of NAACLHLT, 1627-1637.

Sutskever, I.; Martens, J.; and Hinton, G. 2011. Generating text with recurrent neural networks. In Proceedings of the 28th International Conference on Machine Learning (ICML-11), 1017-1024.

Upadhyay, S.; Faruqui, M.; Dyer, C.; and Roth, D. 2016. Cross-lingual models of word embeddings: An empirical comparison. In Proceedings of ACL, 1661-1670.

Wang, D.; Cui, P.; and Zhu, W. 2016. Structural deep network embedding. In Proceedings of KDD, 1225-1234. 